\title{
An Efficient Image Compression Approach based on DWT with Quality Parameter (PSNR)
}

\author{
Babul Bijan Mandal \\ M.Tech Scholar \\ Computer Science Engineering \\ Department
}

\author{
Gyander Singh Tomar \\ Asst. Professor \\ Dr. MPS Group of Institution, Agra
}

\author{
Vedant Rastogi \\ Associated Professor \\ Computer Science Engineering \\ Department
}

\begin{abstract}
The overall objective of performing a set of instruction with wavelets to identify the impact of wavelets algorithms on optimization approaches by scope, performance and cost. This Self Literature Review is conducted on more than 20 articles, and develops the algorithms for MATLAB using wavelets and optimized the algorithms with respect to MSE, PSNR and bpp.
\end{abstract}

\section{Keywords}

Image compression, wavelets, storage, etc

\section{INTRODUCTION}

With advances in multimedia technologies, demand for transmission and storage of voluminous multimedia data has dramatically increased and, as a consequence, data compression is now essential in reducing the amount of data prior storage or transmission. Compression techniques aim to minimise the number of bits required to represent image data while maintaining an acceptable visual quality. Image compression is achieved by exploiting the spatial and perceptual redundancies present in image data. Image compression techniques are classified into two categories, lossless and lossy. Lossless techniques refer to those that allow recovery of the original input data from its compressed representation without any loss of information, i.e. after decoding, an identical copy of the original data can be restored. Lossy techniques offer higher compression ratios but it is Impossible to recover the original data from its compressed data, as some of the input information is lost during the lossy compression. These techniques are designed to minimize the amount of distortion introduced into the image data at certain compression ratios. Compression is usually achieved by transforming the image data into another domain, e.g. frequency or wavelet domains, and then quantizing and losslessy encoding the transformed coefficients (Ghanbari, 1999; Peng \& Kieffer, 2004; Wang et al., 2001). In recent years much research has been undertaken to develop efficient image compression techniques. This systems and advanced multimedia applications. The objective of this paper is to evaluate a set of wavelets for image compression. Image compression using wavelet transforms results in an improved compression ratio. Here in this paper we examined and compared various wavelet families such as Haar, Symlets and Biorthogonal using Discrete Wavelet Transform and Fast wavelet transform. In DWT wavelets are discretely sampled. The Discrete Wavelet Transform analyzes the signal at different frequency bands with different resolutions by decomposing the signal into an approximation and detail information. The study compares DWT and FWT approach in terms of PSNR, Compression Ratios and elapsed time for several Images. Complete analysis is performed at second and third level of decomposition using Haar Wavelet, Symlets wavelet and Biorthogonal wavelet. The research has led to the development of two standard image compression techniques called: JPEG and JPEG2000 (JPEG, 1994; JPEG 2000, 2000), and many nonstandard image compression algorithms (Said \& Pearlman, 1996; Scargall \& Dlay, 2000; Shapiro, 1993).

\section{SURVEY}

Image Compression Using Hybrid DCT-DWT Transform, 2015 K. Ayyappa Swamy [1] presents image compression using Discrete Cosine Transform (DCT) for different quantization laves we can observe different compression ratio and different peak signal-to-noise ratio. In Discrete Wavelet Transform (DWT) , with different levels and different thresholds we can observe different compression ratio and different peak signal-to-noise ratio. Based on the application we can select the quantization level. When comparing DCT with DWT, results of DWT are better than DCT. In this paper we are proposing a new hybrid transform by combining DCT and DWT which gives better compression ratio for same PSNR. In proposed transform first image is compressed using DWT and the approximation coefficients of compressed image are again compressed using DCT.

Varinderjit Kaur [2] presents Image compression is application of reducing the size of graphics file, without compromising on its quality. Depending on the reconstructed image, to be exactly same as the original or some unidentified loss may be incurred, two type of techniques for compression exist. Two techniques are: one is lossy techniques and other is lossless techniques. Image compression is the application of Data compression on digital images. Data compression is the technique to reduce the redundancies in data representation in order to decrease data storage requirements and hence communication costs. Reducing the storage requirement is equivalent to increasing the capacity of the storage medium and hence communication bandwidth. Thus the development of efficient compression techniques will continue to be a design challenge for future communication

implementation of the proposed algorithm based on video watermarking us used Matlab software. The implementation is done under the Image Processing Toolbox in the Matlab.

D. Ravichandran presents [3] hybrid algorithmic approach for medical image compression based on multilevel decomposition using Haar wavelet transform and Huffman variable entropy coding and the image reconstruction is done with wavelet packet. The experimental results show that the proposed hybrid algorithmic approach provides high compression ratio, least mean square error (MSE) and better Peak Signal to Noise ratio (PSNR) between original and reconstructed image. There are various possible directions for future investigations using evolutionary computation such as Fuzzy Logic, Genetic Algorithms (GA) and Artificial Neural 
Networks (ANN) in order to optimize compression techniques.

Somasundar Reddy et al. [29] proposed a theory based on Image compression which is of prime importance in Real time applications like video conferencing where data are transmitted through a channel. This method provides better results in terms of compression ratio, mean square error and peak signal to noise ratio. Proposing a simple and effective method combined with effective thresholding for further compression in this paper that saves a lot of bits in the image data transmission. There are very wide ranges of practical value for today that have a large number of image data to be transmitted.

Fathima et al. [30] analyzed lossy compression of an RGB image by 2D Haar DWT and recovered it by inverse operation and provide acceptable recovery at a glance till $90 \%$ of zeros on each block. The algorithm is much simpler than JPEG or JPEG 2000 hence the technique is applicable in pattern recognition of complicated image like biometric (human face, fingerprint, retina or manual signature) identification where the process time is the main concern. The entire work can be extended using other wavelet matrix to make the comparison of performance even we can apply the algorithm for de-nosing operation of images.

\section{PROBLEM STATEMENT}

As a user of graphics file conversion applications we have been interested in this field since our interest in computing began. Our own experiences of using images for compression for course-work has led us to think about many questions as to why there are so many algorithms and methods for storing and transferring images in a compressed form. This project has given us the opportunity to find out the answers to our questions.

The learning curve embarked on has been considerably steeper than previous work we have undertaken. The software component constitutes our first true software development culminating in a final product. Our previous knowledge of the MATLAB did not outfit for the scale of this work, and our skills in other languages were only of a basic level. Through the development we have learnt everything necessary about this language and how they can be applied to creating image compression software.

\section{QUALITY PARAMETER}

\section{a) Mean Square Error}

Benchmarks in image data compression are the compression ratio and PSNR (Peak Signal to Noise Ratio). The compression ratio is used to measure the ability of data compression by comparing the size of the image being compressed to the size of the original image. The greater the compression ratio means the better the wavelet function. PSNR is one of the parameters that can be used to quantify image quality. PSNR parameter is often used as a benchmark level of similarity between reconstructed image and the original image. Larger PSNR will produce better image quality.

The Mean Square Error (MSE) is the error metrics used to compare image compression quality. The MSE represents the cumulative squared error between the compressed and the original image, whereas PSNR represents a measure of the peak error. The lower the value of MSE, the lower the error.

$$
M S E=\frac{\sum_{M, N}\left[I_{1}(m, n)-I_{2}(m, n)\right]^{2}}{M^{*} N}
$$

In the previous equation, $M$ and $N$ are the number of rows and columns in the input images, respectively.

When the pixels are represented using 8 bits per sample, this is 255. More generally, when samples are represented using linear PCM with B bits per sample, MAXI is $2 \mathrm{~B}-1$. For color images with three RGB values per pixel, the definition of PSNR is the same except the MSE is the sum over all squared value differences divided by image size and by three. Typical values for the PSNR in lossy image and video compression are between 30 and $50 \mathrm{~dB}$, where higher is better[6] Acceptable values for wireless transmission quality loss are considered to be about $20 \mathrm{~dB}$ to $25 \mathrm{~dB}$ [8].

\section{b) Compression Ratio}

The compression ratio is equal to the size of the original image divided by the size of the compressed image. This ratio gives an indication of how much compression is achieved for a particular image. Most algorithms have a typical range of compression ratios that they can achieve over a variety of images. Because of this, it is usually more useful to look at an average compression ratio for a particular method.

The compression ratio typically affects the picture quality. Generally, the higher the compression ratio, the poorer the quality of the resulting image. The tradeoff between compression ratio and picture quality is an important one to consider when compressing images. Furthermore, some compression schemes produce compression ratios that are highly dependent on the image content. For example, a highly detailed image of a crowd at a football game may produce a very small compression ratio, whereas an image of a pure blue sky may produce a very high compression ratio.

Thus a gray scale image when displayed will have shades of gray, ranging from 0 (black) to 255 (white). An uncompressed, say $800 \times 800$ pixel image, will need 64 x 104, bytes $\sim 0.64 \mathrm{MB}$. If 1000 images are to be stored, we need 640 MBL Obviously, we then need to compress the image, i.e. represent the same image with reduced number of bits, possibly with some loss, without changing the original size of the image. Mathematically, a measure of compression is given by the compression ratio (C R) defined as, number of bits in the original image R-(1) number of bits in the compressed image while compressing an image, two important objectives are kept in mind. On one hand, the compressed image should not be distorted and on the other, it should require minimum number of bytes to store. Typically these two objectives are conflicting, thus a suitable criterion is needed to reach a compromise. This criterion depends upon the particular application. As will be seen soon, it is possible to have a visually pleasing image quality, with a highly lossy compression [13]

\section{c) Rate}

A digital image can be represented by a two dimensional (2D) array i.e., a matrix, each of whose element $J(i, j)$ corresponds to the value of the $(i, j)$ th pixel in then image. If each pixel represents a shade of gray in monochrome images, we need to allocate only one byte or 8bits per pixel (bpp). With $28=256$ combinations, one can represent numbers ranging from 0 to 255 [14].

In the imaging context, the pixel or block of pixel is consider as a set of symbol. A code, on the other hand, is sequence of 
symbols or number that are used to represent information. A string of codes is called a code word. The whole data compression process can now we visualized as a mapping of all possible sequence of symbols of a message (of N1 bits), separately or in a file, to a set of codes, separately or infield, using $\mathrm{N} 2$ bits. The compression ratio(Cr) is then defined as $\mathrm{N} 1 / \mathrm{N} 2$ and the relative redundancy id defined as

\section{$\mathrm{Rd}=1-1 / \mathrm{Cr}$}

Now these scenarios emerge. In scenario one, $\mathrm{N} 2=\mathrm{N} 1$, this means that the compression ratio is 1 and relative redundant is $1-1 / 1=0$. This indicates that there is no redundancy in the image. The input message is reproduces exactly.

\section{PROPOSED ALGORITHMS:}

Step 1: Read input image.

Step 2: Wavelets decomposition

Step 3: Encoding Start

Step 4: Decoding

Step 5: Inverse wavelets decomposition

Step 6: Calculate bpp

Step 7: Calculate MSE

Step 8: Calculate PSNR

\section{INPUT IMAGE}

Using above proposed algorithms designed a tool in MATLAB and run on MATLAB command, with different test image which is shown in below figure.

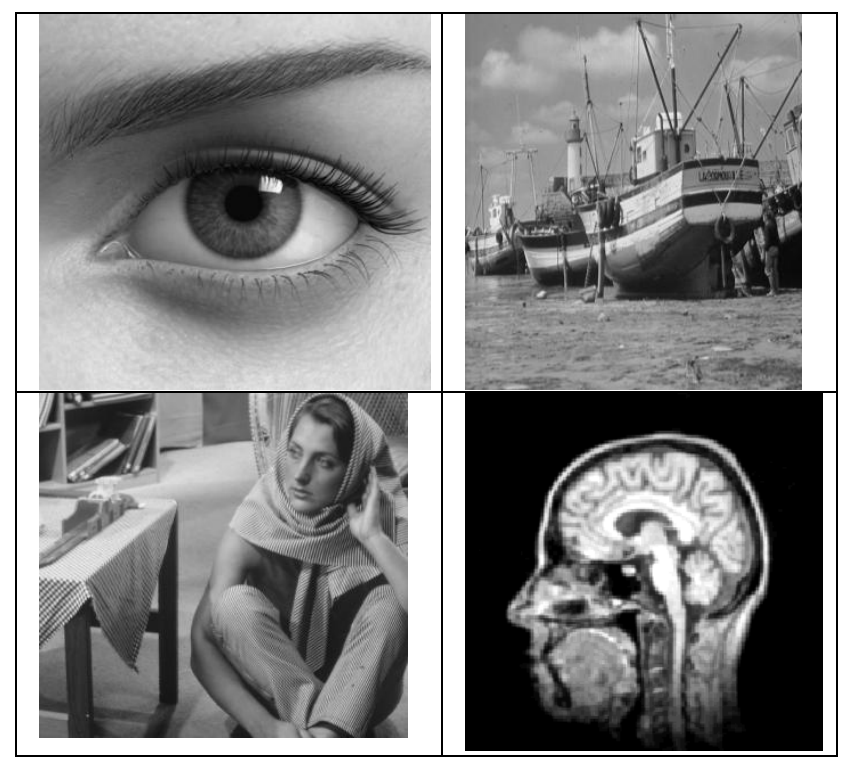

Figure 1: Test Image

\section{RESULT}

In order to evaluate the performance of the proposed method with four input image experiments were performed. In the sets of experiments the performance of the proposed algorithms were recorded with respect to mse, psnr and bit per pixel (bit rate) with same threshold . In below screen shot of matlab command window results are shown with bit rate $0.07 \mathrm{bpp}$ with threshold 50 , mse is 53.9653 and psnr is 30.81 .

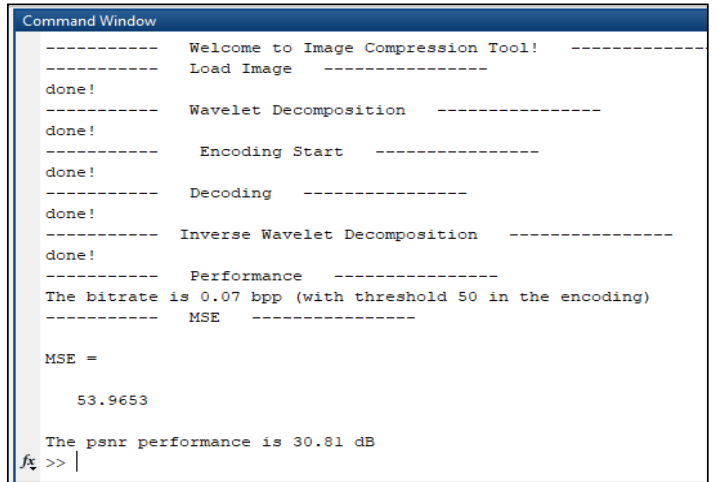

Figure2: MATLAB result

Table 1: Result with input Image

\begin{tabular}{|l|l|l|l|l|l|}
\hline Sno & $\begin{array}{l}\text { Test } \\
\text { Image }\end{array}$ & Threshold & Bpp & PSNR & MSE \\
\hline 1 & $1 . b m p$ & 50 & .33 & 22.93 & 330.9502 \\
\hline 2 & $2 . b m p$ & 50 & .07 & 30.81 & 53.9653 \\
\hline 3 & $3 . b m p$ & 50 & .26 & 27.86 & 106.440 \\
\hline 4 & $4 . b m p$ & 50 & .22 & 26.56 & 143.4766 \\
\hline
\end{tabular}

\section{COMPARISON}

The PSNR measurements for the test images at different bpp using the proposed algorithms are shown in table 1. It is well known that the PSNR is reliable metric for measuring the visual quality of the decompressed images.

The Peak-Signal-to-Noise-Ratios (PSNR) for average bpp shown in Table 2 for all the testing images with proposed algorithms. We can find that the mean PSNR of proposed algorithms is high with OCAO methods [9] . The proposed method is the highest of all methods at $0.25 \mathrm{bpp}$.

Table 2: Average PSNR with bpp

\begin{tabular}{|l|l|l|l|}
\hline Sno & Method & Average Bpp & $\begin{array}{l}\text { Average } \\
\text { PSNR }\end{array}$ \\
\hline 1 & $\begin{array}{l}\text { Proposed } \\
\text { Method }\end{array}$ & 0.22 & 27.04 \\
\hline 2 & QCAO [9] & 0.25 & 26.79 \\
\hline
\end{tabular}

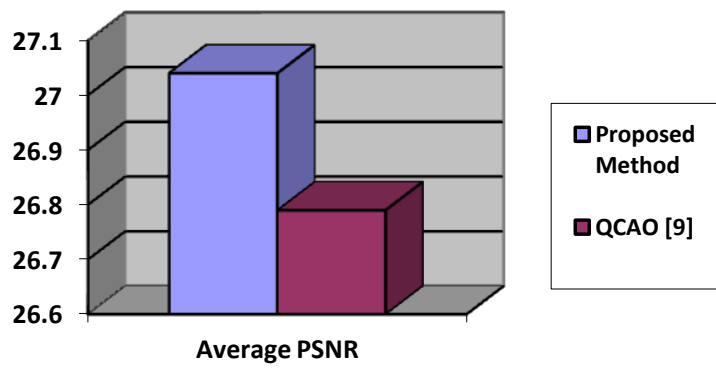

\section{CONCLUSION}

Experimental results showed that the proposed coding scheme provides significantly higher subjective and objective quality when perceptual weights are used to regulate the threshold values. The results also indicated that the proposed method is better than the other method (QCAO) in the respect of bpp. The value of PSNR should be maximum for minimum error between original image and decompressed image. In our 
method average PSNR value is 27.04 which is higher than the QCAO i.e 26.79.

\section{REFERENCES}

[1] Mahinderpal Singh, Meenakshi Garg "Mixed DWT-DCT Approched Based Image Compression Technique" International Journal Of Engineering And Computer Science ISSN:2319-7242 Volume 3 Issue 11 November, 2014 Page No. 9008-9111.

[2] K. Ayyappa Swamy , C. Somasundar Reddy, K. Durga Sreenivas, Image Compression Using Hybrid DCTDWT Transform, Volume 5, Issue 5, May 2015 ISSN: $2277128 \mathrm{X}$

[3] Varinderjit Kaur*, Anamika Pathania, Er.Kiran Bala, "A Review Paper On Advance Digital Image Compression Using Fast Wavelet Transforms Comparative Analysis With Dwt”, [Kaur, 4(3): March, 2015]ISSN: 2277-9655.

[4] Prof D. Ravichandran, Ashwin Dhivakar M R, Dr. Vijay Dakha, "Hybrid Algorithmic Approach for Medical Image Compression Based on Discrete Wavelet Transform (DWT) and Huffman Techniques for Cloud Computing", International Journal of Advanced Engineering, Management and Science (IJAEMS) [Vol1, Issue-3, June- 2015] ISSN: 2454-1311 .

[5] C.SomasundarReddy, "Image Compression using Complex Wavelet Transform (CWT) with Custom Thresholding" 978-1-5090-5686-6/17 /IEEE 2017.

[6] Fahima Tabassum, Md. Imdadul Islam "A Simplified Image Compression Technique Based on Haar Wavelet Transform",978-1-4673-6676-2115/ IEEE 2015.

[7] C.SomasundarReddy, "Image Compression using Complex Wavelet Transform (CWT) with Custom Thresholding" 978-1-5090-5686-6/17 /IEEE 2017.

[8] Fahima Tabassum, Md. Imdadul Islam "A Simplified Image Compression Technique Based on Haar Wavelet Transform",978-1-4673-6676-2115/ IEEE 2015.

[9] Hui Liua, Ke-Kun Huanga, $\square$, Chuan-Xian Renb, Yu-Feng Yub, Zhao-Rong Laic, "Quadtree coding with adaptive scanning order for space-borne image Compression",
Elsevir, Signal Processing: Image Communication 55 (2017) 1-9

[10] Akshay Kekre , Dr. Sanjay Pokle "Improved Image Compression Using Wavelet Transform and Differential Pulse Code Modulation Technique" International Journal of Engineering Research \& Technology (IJERT) Vol. 2 Issue 7, July - 2013.

[11] Neelesh Kumar Sahu,ChandrashekharKamargaonkar "A Survey on Various Medical Image Compression Techniques" International Journal of Science, Engineering and Technology Research (IJSETR) Volume 2, Issue 2, February 2013.

[12] NavpreetSaroya,PrabhpreetKaur "Analysis Of Image Compression Algorithm Using DCT And DWT Transforms" International Journal of Advanced Research in Computer Science and Software Engineering 4(2), February - 2014, pp. 897-900.

[13] R. Bhavithra, L. AyeeshaBegame, K.S.L. Deepika "A Survey on Medical Image Compression Based on Transform "SSRG International Journal of Electronics and Communication Engineering (SSRG-IJECE) volume1 issue6 August 2014.

[14] MalvikaDixit,Harbinder Singh "An Enhanced Hybrid Technology for Digital Image Compression "SSRG International Journal of Electronics and Communication Engineering (SSRG-IJECE) - volume1 issue7 Sep 2014.

[15] MahinderpalSingh,MeenakshiGarg "Mixed DWT-DCT Approached Based Image Compression Technique" International Journal Of Engineering And Computer Science ISSN: 2319-7242 Volume 3 Issue 11 November, 2014 Page No. 9008-9111.

[16] K. Ayyappa Swamy , C. Somasundar Reddy, K. Durga Sreenivas , Image Compression Using Hybrid DCTDWT Transform, Volume 5, Issue 5, May 2015 ISSN: $2277128 \mathrm{X}$

[17] Kumar, T. and K. Verma, 2010a. "A Theory Based On Conversion Of RGB Image To Gray Image".IJCA, 7: 512. DOI: $10.5120 / 1140-1493$. 